\title{
Fungos micorrízicos arbusculares e adubação fosfatada em mudas de mangabeira
}

\author{
Cynthia Maria Carneiro Costa ${ }^{(1)}$, Uided Maaze Tiburcio Cavalcante(1), Bruno Tomio Goto(1), \\ Venézio Felipe dos Santos ${ }^{(2)}$ e Leonor Costa Maia ${ }^{(1)}$
}

\begin{abstract}
(1)Universidade Federal de Pernambuco, Centro de Ciências Biológicas, Dep. de Micologia, Av. Prof. Nelson Chaves, s/no, Cidade Universitária, CEP 50670-420 Recife, PE. E-mail: cynthiamccosta@hotmail.com, umaaze@hotmail.com.br, brunogoto@hotmail.com, leonorcmaia@yahoo.com.br (2)Empresa Pernambucana de Pesquisa Agropecuária, Av. Gal. San Martin 1371, CEP 50761-000 Recife, PE.
\end{abstract}

\begin{abstract}
Resumo - O objetivo deste trabalho foi avaliar os efeitos de fungos micorrízicos arbusculares (FMA) e da adubação fosfatada em mudas de mangabeira (Hancornia speciosa Gomes). O experimento, em casa de vegetação, utilizou delineamento inteiramente casualizado em fatorial com dois tratamentos de solo nativo oriundo de pomar com mangabeiras, desinfestado com brometo de metila e não-desinfestado, seis doses de $\mathrm{P}\left(3^{*}, 3,48,93\right.$, 138 e $183 \mathrm{mg} \mathrm{dm}^{-3}$ ) e três tratamentos de inoculação, Gigaspora albida Schenck \& Smith, Glomus etunicatum Becker \& Gerdemann e controle sem inoculação, com quatro repetições. O tratamento $3 *$ não recebeu solução nutritiva e os demais receberam solução nutritiva de Hoagland sem fósforo por ocasião da inoculação. Após 150 dias, observou-se aumento na altura, biomassa e área foliar nos tratamentos com G. albida, em solo desinfestado. Respostas à inoculação ocorreram nas mudas cultivadas com a menor dose de $\mathrm{P}$, nos dois tratamentos de solo. A mangabeira mostrou-se dependente da micorrização apenas na menor dose de $\mathrm{P}$ em solo desinfestado. Nos demais níveis de P, a dependência variou em função do FMAe da condição do solo. A associação com G. albida proporcionou melhor desenvolvimento das mudas de mangabeira.
\end{abstract}

Termos para indexação: Hancornia speciosa, associação micorrízica, dependência micorrízica, fruteira.

\section{Arbuscular mycorrhizal fungi and phosphorus supply on seedlings of mangabeira}

\begin{abstract}
The objective of this work was to evaluate the effects of the association of arbuscular mycorrhizal fungi (AMF) and phosphorus fertilization on seedlings of "mangaba" (Hancornia speciosa). The experiment, at a greenhouse, was in a completely randomized factorial design with two treatments of the native soil from an H. speciosa orchard, fumigated with methyl bromite and non-fumigated, six doses of $\mathrm{P}\left(3^{*}, 3,48,93,138\right.$ and $183 \mathrm{mg} \mathrm{dm}^{-3}$ ) and three inoculation treatments, Gigaspora albida Schenck \& Smith, Glomus etunicatum Becker \& Gerdemann and an uninoculated control, with four replicates. The treatment $3 *$ did not receive nutrient solution and the others received Hoagland nutrient solution without phosphorus, at the time of inoculation. After 150 days, high increment on height, shoot biomass and leaf area were observed in the treatments with G. albida, in fumigated soil. Positive response to inoculation occurred in seedlings cultivated in soil with the lower phosphorus level, in both soil treatments. Hancornia speciosa was mycorrhiza dependent only when in fumigated soil, at the lowest phosphorus level. In treatments at other levels, the dependency varied according to the AMF and soil condition. The association with G. albida enhanced growth of H. speciosa.
\end{abstract}

Index terms: Hancornia speciosa, mycorrhizal association, mycorrhizal dependency, fruit tree.

\section{Introdução}

A mangabeira - Hancornia speciosa Gomes - é uma fruteira nativa do Brasil e apresenta frutos com sabor peculiar, sendo $80 \%$ da polpa aproveitada na fabricação de sucos, sorvetes, doces, vinhos, vinagres, licores e xaropes, além do consumo in natura. No entanto, a falta de informações sobre essa cultura vem restringindo seu cultivo comercial, tornando o aproveitamento limitado a pequenos produtores e à exploração extrativista (Aguiar Filho et al., 1998; Donadio et al., 2002).

Conhecimentos técnicos relativos à propagação, desenvolvimento, exigências hídricas e nutricionais, adubação e práticas culturais são imprescindíveis na exploração racional de uma espécie nativa. Os fungos micorrízicos arbusculares (FMA) podem ser utilizados como biofertilizantes naturais na produção de mudas, pois, nesse estádio de formação, a inoculação pode ga- 
rantir o sucesso do estabelecimento da simbiose, reduzindo a necessidade de aplicação de insumos químicos, como verificado em aceroleiras (Costa et al., 2001), maracujazeiro-amarelo (Cavalcante et al., 2002a) e bananeiras (Declerck et al., 2002).

A dependência micorrízica de fruteiras tropicais tem sido alvo de estudos (Trindade et al., 2000; Cavalcante et al., 2001), por serem culturas que podem produzir ótimos resultados.

O objetivo deste trabalho foi avaliar os efeitos de fungos micorrízicos arbusculares (FMA) e da adubação fosfatada em mudas de mangabeira.

\section{Material e Métodos}

O solo utilizado no experimento foi coletado $(0-20 \mathrm{~cm})$ em pomar natural de mangabeira, localizado no Município de Sirinhaém, PE e apresentou as seguintes características: classe textural arenoso; $\mathrm{pH}, 5,4 ; 3 \mathrm{mg} \mathrm{dm}^{-3}$ de P; 0,50, 0,60, 0,70 e 0,03 cmol $_{\mathrm{c}} \mathrm{dm}^{-3}$ de Al, Ca, Mg e $\mathrm{K}$, respectivamente. A partir de amostras compostas, cada uma de quatro subamostras, da rizosfera de dez mangabeiras, esporos de FMA foram extraídos por peneiramento úmido (Gerdemann \& Nicolson, 1963) e centrifugação em água e sacarose (Jenkins, 1964), montados em lâminas e identificados (Schenck \& Pérez, 1990; West Virgínia University, 2003).

O experimento foi conduzido em delineamento inteiramente casualizado, em fatorial de $2 \times 6 \times 3$, sendo dois tratamentos de solo, desinfestado ou não com brometo de metila, seis doses de $\mathrm{P}\left(3,3^{*}, 48,93,138\right.$ e $\left.183 \mathrm{mg} \mathrm{dm}^{-3}\right)$ e três tratamentos de inoculação, Gigaspora albida Schenck \& Smith - UFPE 01, Glomus etunicatum Becker \& Gerd. - UFPE 08, e controle sem inoculação, com quatro repetições, totalizando 144 potes. A definição das doses de P foi baseada em ensaios prévios, quando a mangabeira não respondeu até $60 \mathrm{mg} \mathrm{dm}^{-3}$ de fósforo. Os níveis de $\mathrm{P}$ usados neste trabalho foram obtidos pela incorporação, ao solo, de fosfato monobásico de potássio $\left(\mathrm{KH}_{2} \mathrm{PO}_{4}\right) \mathrm{em}$ solução. Com exceção do tratamento $3^{*}$, os demais receberam solução nutritiva de Hoagland isenta de P, por ocasião da inoculação.

Os isolados de FMA foram multiplicados em associação com painço (Panicum milliacium L.) e os esporos extraídos do solo para serem utilizados como inoculante. $\mathrm{Na}$ obtenção das mudas, sementes de frutos maduros foram despolpadas com auxílio de calcário dolomítico, lavadas em água corrente, secadas e semeadas no mesmo dia. Quando as plantas apresentavam dois pares de folhas não-cotiledonares, foram transplantadas para sacos pretos de polietileno com $4,5 \mathrm{~kg}$ de solo e submeti- das à inoculação de 200 esporos de FMA em suspensão/pote. Durante o período experimental, a temperatura e a umidade relativa do ar, máximas e mínimas, fo$\operatorname{ram} 34,1^{\circ} \mathrm{C}$ e $82 \%$ e $22^{\circ} \mathrm{C}$ e $45 \%$, respectivamente.

Após 150 dias, foram avaliados altura, área foliar, biomassa seca da parte aérea, concentração de $\mathrm{P}$ na parte aérea, colonização por arbúsculos, hifas e colonização total e o incremento promovido pela inoculação. $\mathrm{O} P$ foi extraído por digestão nitro-perclórica e quantificado em espectrofotômetro (Malavolta, 1989). A área foliar foi calculada pelo Programa SIARCS 3.0 da Embrapa (Jorge et al., 1996). As raízes foram clarificadas com KOH (Phillips \& Hayman, 1970), lavadas e acidificadas com $\mathrm{HCl} 1 \%$ por três minutos, coradas com azul de Trypan 0,05\% e avaliadas (McGonigle et al., 1990). O incremento, em relação ao controle, foi calculado pela fórmula de Edigiton et al. (1971) adaptada: $\mathrm{I}(\%)=[(\mathrm{Tr}-\mathrm{T}) / \mathrm{T}] 100$, em que I $(\%)$ é o incremento da variável, Tr é o valor médio para o tratamento micorrizado e T é o valor médio do controle sem inoculação.

A dependência micorrízica relativa (DMR) da mangabeira foi calculada segundo Plenchette et al. (1983). Os graus de dependência estabelecidos, usando as categorias propostas por Habte \& Manjunath (1991), foram modificados, considerando-se os valores obtidos em solo com os diversos níveis de fósforo. Os dados foram submetidos à análise de variância, as médias comparadas pelo teste de Tukey a 5\% de probabilidade e as análises de regressão feitas pelo programa SANEST.

\section{Resultados e Discussão}

$\mathrm{Na}$ rizosfera da área estudada, foram identificadas 13 espécies de FMA, representantes de quatro famílias de Glomeromycota: Acaulosporaceae: Acaulospora foveata Trappe \& Janos, A. longula Spain \& Schenck, A. mellea Spain \& Schenck, A. scrobiculata Trappe, A. tuberculata Janos \& Trappe, Entrophospora colombiana Spain \& Schenck; Archaeosporaceae: Archaeospora leptoticha (Schenck \& Smith) Morton \& Redecker; Gigasporaceae: Gigaspora gigantea (Nicol. \& Gerd.) Gerd. \& Trappe; e Glomaceae: Glomus etunicatum Becker \& Gerd., G. fuegianum (Spegazzini) Trappe \& Gerd., G. glomerulatum Sieverding, G. macrocarpum Tul. \& Tul. e Glomus sp. Dentre essas, Glomus fuegianum constitui novo registro de ocorrência em solos brasileiros. Acaulospora mellea, A. scrobiculata, A. tuberculata, E. colombiana, G. gigantea e G. etunicatum foram citadas anteriormente em solos de cerrado (Miranda \& Miranda, 1997), tipo de vegetação ao qual a mangabeira está associada. 
Em solo desinfestado, apenas as mudas associadas com G. albida apresentaram respostas significativas no crescimento. Quando cultivadas no menor nível de P, apresentaram maior altura e biomassa seca da parte aérea do que as cultivadas em solo com as demais doses de fósforo. Entre os tratamentos de inoculação, a associação com G. albida também proporcionou maior altura e biomassa seca da parte aérea do que os demais tratamentos (Tabela 1).

O crescimento do maracujazeiro-amarelo também foi favorecido pela inoculação de G. albida (Cavalcante et al., 2002a). Em genótipos de aceroleira, a simbiose com G. margarita proporcionou maior altura e biomassa seca da parte aérea, em solo esterilizado e com $4 \mathrm{mg} \mathrm{dm}^{-3}$ de P no solo (Costa et al., 2001). Respostas semelhantes com G. margarita foram observadas em mudas de goiabeira que apresentaram aumento no crescimento na menor dose de P (Samarão \& Martins, 1999) e em bananeiras micropropagadas, onde o efeito benéfico desse FMA foi modulado pelo substrato de crescimento (Lins et al., 2003).

Em geral, a associação com G. etunicatum não promoveu o crescimento da mangabeira em relação ao controle (Tabela 1). O mesmo foi observado em limoeiro-cravo, cujo tratamento com G. etunicatum não diferiu do controle (Melloni et al., 2000). Esses resultados demonstram, mais uma vez, a influência dos genótipos dos simbiontes na eficiência da simbiose.
No solo não-desinfestado, diferenças significativas na altura e biomassa seca da parte área, em relação aos níveis de $\mathrm{P}$, foram observadas apenas nas mudas associadas com G. etunicatum, cultivadas em solo com 3 e $48 \mathrm{mg} \mathrm{dm}^{-3}$ de $\mathrm{P}$, com maiores valores no tratamento com baixo fósforo (Tabela 1). Entre os tratamentos de inoculação, registrou-se diferença apenas nas mudas mantidas em solo com $48 \mathrm{mg} \mathrm{dm}^{-3}$ de P; mudas do tratamento controle apresentaram maior altura do que as mudas com G. etunicatum; esses dois tratamentos não diferiram daquele com G. albida. Quanto à biomassa, nenhuma diferença foi observada (Tabela 1).

Em geral, as mudas apresentaram maior altura e biomassa nos tratamentos em solo não-desinfestado, independentemente da inoculação, o que demonstra a eficiência das espécies de FMA nativos, existentes no pomar de mangabeira e cujo solo foi utilizado no experimento. Benefícios também foram observados em cafeeiros submetidos à inoculação de isolados nativos de G. etunicatum procedentes da rizosfera dessas plantas (Saggin Júnior et al., 1994). Comportamento contrário foi constatado em mudas de maracujazeiro-amarelo, em que a desinfestação do solo proporcionou melhores respostas de crescimento (Cavalcante et al., 2002b). Em gravioleiras, os FMA nativos apresentaram efeito antagônico a $S$. heterogama, interação sinérgica com Entrophospora colombiana, e não influenciaram a

Tabela 1. Efeito da desinfestação do solo, da associação com fungos micorrízicos arbusculares e doses de fósforo na altura e biomassa seca da parte aérea de mudas de mangabeira, 150 dias após a inoculação ${ }^{(1)}$.

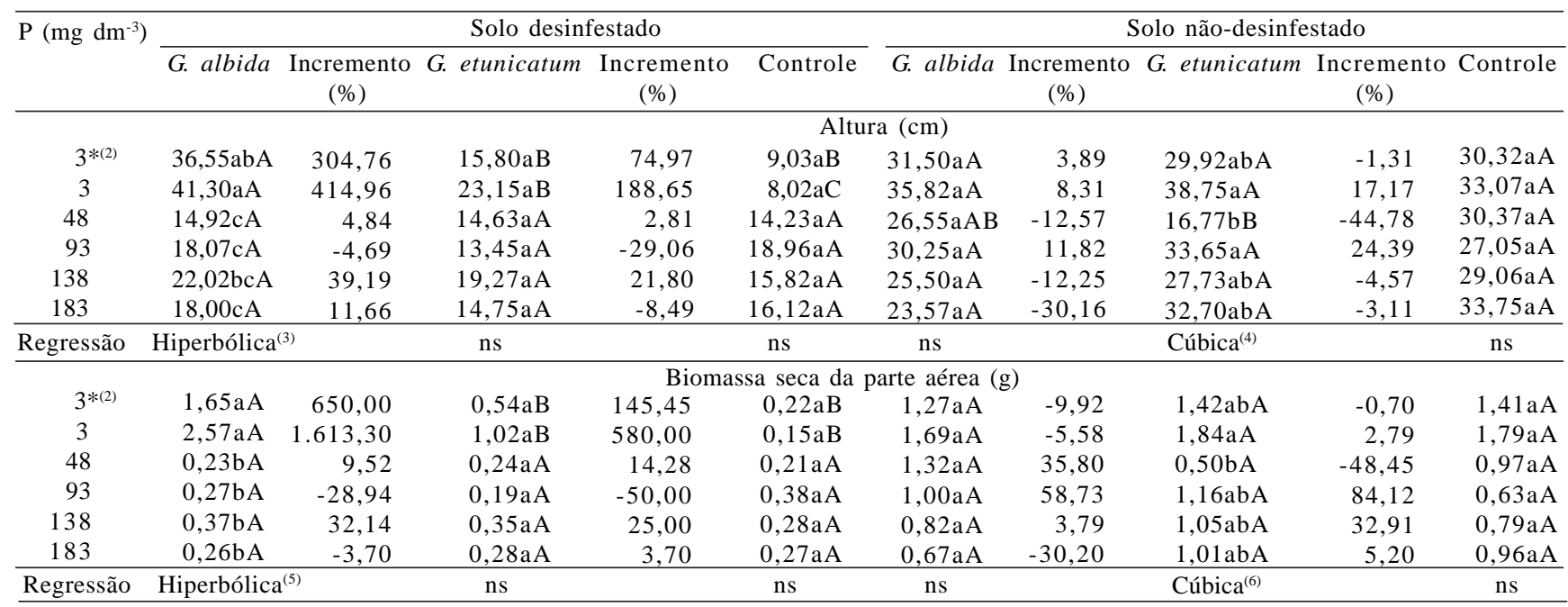

${ }^{(1)}$ Médias seguidas pela mesma letra, minúscula na coluna e maiúscula na linha, não diferem entre si pelo teste de Tukey a 5\% de probabilidade; os coeficientes de variação em relação à altura e à biomassa das mudas de mangabeira foram de 31,91\% e 64,45\%, respectivamente. (2)Isento de solução nutritiva. ${ }^{(3)} \mathrm{y}=17,51^{* *}+70,83^{* *} / \mathrm{x}\left(\mathrm{R}^{2}=0,92\right) .{ }^{(4)} \mathrm{y}=39,95^{* *}-0,76^{*} \mathrm{x}+0,009^{*} \mathrm{x}^{2}-0,00002^{\mathrm{ns}} \mathrm{x}^{3}\left(\mathrm{R}^{2}=0,53\right) .{ }^{(5)} \mathrm{y}=0,2066^{\mathrm{ns}}+7,0811^{* *} / \mathrm{x}$ $\left(\mathrm{R}^{2}=0,99\right) .{ }^{(6)} \mathrm{y}=1,95^{* *}-0,051 \mathrm{x}^{* *}+0,00058^{* *} \mathrm{x}^{2}-0,000001^{*} \mathrm{x}^{3}\left(\mathrm{R}^{2}=0,79\right) .{ }^{\text {ns Não-significativo. }}{ }^{*} \mathrm{e}^{* *}$ Significativo a $5 \%$ e a $1 \%$ de probabilidade pelo teste $\mathrm{t}$, respectivamente. 
eficiência de G. margarita (Chu et al., 2001). $\mathrm{O}$ comportamento do fungo introduzido no solo sem desinfestação depende da sua competitividade com os FMA nativos; infectividade, densidade de inóculo, rapidez no estabelecimento e habilidade em manter o nível de colonização são alguns dos fatores que contribuem para melhor desempenho em condição de competitividade (Wilson \& Tommerup, 1992).

Em solo desinfestado, a inoculação de FMA promoveu incrementos, em relação ao controle sem inoculação, principalmente nos tratamentos em solo com baixo nível de $\mathrm{P}\left(3 \mathrm{mg} \mathrm{dm}^{-3}\right)$, em todos os parâmetros de crescimento das mudas. A altura e a biomassa seca da parte aérea foram influenciadas pelas doses de $\mathrm{P}$ e tratamentos de solo (Tabela 1), mas os incrementos na área foliar foram afetados apenas pelos tratamentos de solo (Tabela 2). Os maiores incrementos na altura $(414,96 \%) \mathrm{e}$ na biomassa seca da parte aérea $(1613,3 \%)$ ocorreram nas mudas colonizadas por G. albida. Mudas associadas a G. etunicatum também apresentaram incremento elevado (Tabela 1).

A colonização do maracujazeiro-amarelo com os mesmos FMA utilizados resultaram em incrementos semelhantes na altura e biomassa seca da parte aérea (Cavalcante et al., 2002b). Em dois genótipos de aceroleira, os incrementos proporcionados pela micorrização variaram de acordo com o FMA e o genótipo estudado (Costa et al., 2001).

Houve interação entre G. albida x P x tratamento de solo, com regressão hiperbólica ajustada para altura e biomassa seca da parte aérea, indicando o crescimento das mudas com inoculação desse fungo, quando cultivadas em solo desinfestado e com as menores doses de fósforo. Nesse caso, a adição de $\mathrm{P}\left(>48 \mathrm{mg} \mathrm{dm}^{-3}\right)$ inibiu a atuação dos FMA, não permitindo que as plantas atin-

Tabela 2. Efeito da desinfestação do solo e da associação com fungos micorrízicos arbusculares (FMA) na área foliar e respectivos incrementos em mudas de mangabeira, independentemente das doses de fósforo, 150 dias após a inoculação ${ }^{(1)}$.

\begin{tabular}{lcclccc}
\hline FMA & \multicolumn{2}{c}{ Solo desinfestado } & & \multicolumn{2}{c}{ Solo não-desinfestado } \\
\cline { 2 - 3 } \cline { 6 - 7 } & $\begin{array}{c}\text { Área foliar } \\
\left(\mathrm{cm}^{2}\right)\end{array}$ & $\begin{array}{c}\text { Incremento } \\
(\%)\end{array}$ & & $\begin{array}{c}\text { Área foliar } \\
\left(\mathrm{cm}^{2}\right)\end{array}$ & $\begin{array}{c}\text { Incremento } \\
(\%)\end{array}$ \\
\hline G. albida & $214,16 \mathrm{aA}$ & 452,24 & & $206,67 \mathrm{aA}$ & $-14,68$ \\
G. etunicatum & $118,68 \mathrm{bB}$ & 206,03 & & $207,66 \mathrm{aA}$ & $-14,27$ \\
Controle $^{(2)}$ & $38,78 \mathrm{cB}$ & - & & $242,23 \mathrm{aA}$ & - \\
\hline
\end{tabular}

${ }^{(1)}$ Médias seguidas pela mesma letra, minúsculas na coluna e maiúsculas na linha, não diferem entre si pelo teste de Tukey a 5\% de probabilidade; o coeficiente de variação foi de 52,52\%. ${ }^{(2)}$ Controle, sem inoculação, utilizado como base para o cálculo do incremento promovido pela inoculação. gissem o máximo crescimento. As regressões cúbicas, ajustadas para altura e biomassa seca da parte aérea foram registradas no tratamento com G. etunicatum, em solo não-desinfestado (Tabela 1). O aumento do $\mathrm{P}$ (>93 $\mathrm{mg} \mathrm{dm}^{-3}$ ) pode ter favorecido a atividade de outros microrganismos promotores de crescimento vegetal presentes no solo. Em relação aos FMA, a combinação dos nativos e introduzidos aparentemente foi benéfica, considerando que não houve inibição do efeito da micorrização com a adição de doses mais elevadas de fósforo. Assim, o crescimento das mudas foi praticamente o mesmo em todos os tratamentos de adubação (Tabela 1).

No solo desinfestado, a área foliar das mangabeiras associadas a G. albida foi maior do que nas plantas em simbiose com G. etunicatum, ambos superiores ao controle (Tabela 2). Tais resultados confirmam os obtidos com aceroleiras, nas quais se estudou o efeito de FMA e observou-se que a simbiose dessa fruteira com G. margarita aumentou a área foliar em relação à associação com G. etunicatum e o controle (Costa et al., 2001). Entretanto, em maracujazeiro-amarelo, não houve diferença na resposta em relação a esses mesmos fungos (Cavalcante et al., 2002b).

No solo natural, não se observou diferença entre os tratamentos de inoculação e, aparentemente, a presença de fungos nativos favoreceu o aumento da área foliar em mudas associadas a G. etunicatum, não inibindo a ação de G. albida. Por outro lado, só se registrou incremento no solo desinfestado nas mudas com inoculação (Tabela 2).

Interações entre P x FMA e solo ocorreram na colonização total, com regressão cúbica em relação às mudas associadas a G. albida e cultivadas em solo desinfestado com baixa dose de fósforo. Em solo nãodesinfestado também houve interação entre $\mathrm{Px}$ G. albida e $\mathrm{P} \times$ controle, com regressões quadráticas e interação entre P x G. etunicatum, com regressão cúbica (Tabela 3). Esse último comportamento pode ter ocorrido devido às interações positivas entre os microrganismos presentes no solo. Em relação aos FMA, a combinação dos nativos e introduzidos aparentemente foi benéfica, considerando que não houve inibição do efeito da micorrização mesmo com a adição de doses mais elevadas de fósforo.

No solo desinfestado, mudas associadas a G. albida apresentaram maior colonização total nos tratamentos com as menores doses de fósforo. Nas mudas com G. etunicatum não houve diferença na colonização total com o aumento das doses de P, o que ocorreu no solo não-desinfestado. Gigaspora albida destacou-se por proporcionar maior colonização, mostrando-se mais 
competitiva que G. etunicatum quando em solo desinfestado. Em solo natural, não houve diferença significativa entre os tratamentos com fungos nativos e introduzidos, em solo não-desinfestado, exceto na dose de $138 \mathrm{mg} \mathrm{dm}^{-3}$ de $\mathrm{P}$ no solo nas mudas com G. etunicatum, em relação às associadas com G. albida (Tabela 3).

Em Phleum pratense L. (Clapperton \& Reid, 1992) e em plantas de alho (Allium porrum L.) (Amijee et al., 1989) a colonização total das raízes foi inibida pela fertilização, assim como a porcentagem de hifas e arbúsculos, sendo a formação de arbúsculos consistentemente menor nas raízes das plantas mantidas em solo fertilizado do que em solo não-fertilizado, embora em alguns casos sem diferenças significativas. Esses resultados concordam com os deste trabalho e reforçam a importância dos arbúsculos na troca de nutrientes entre o fungo e a planta. A presença dessas estruturas é fundamental no funcionamento da simbiose micorrízica (Clapperton \& Reid, 1992).

Acompanhando a tendência observada na área foliar, nos tratamentos com solo não-desinfestado houve maior concentração de $\mathrm{P}$ na parte aérea, maior porcentagem de arbúsculos e de hifas, fazendo com que G. albida produzisse mais hifas que G. etunicatum e os FMA nativos. Entretanto, com a desinfestação do solo, a atuação de G. albida foi melhor observada, destacando-se em relação aos outros tratamentos de inoculação em todos os parâmetros, exceto porcentagem de hifas.

Os FMA nativos competiram eficientemente com os fungos introduzidos, colonizando o hospedeiro com a mesma intensidade (Tabela 4). A efetividade da população nativa de FMA também foi demonstrada pelo aumento no conteúdo de $\mathrm{P}$ na parte aérea de bananeiras (Declerck et al., 2002) e gravioleiras (Chu et al., 2001). No entanto, o mesmo não ocorreu em mudas de maracujazeiro-amarelo (Cavalcante et al., 2002a).

Nos tratamentos com solo desinfestado, G. etunicatum colonizou fracamente o hospedeiro, como comprovado pela porcentagem de arbúsculos e hifas formadas nas raízes, que não diferiu significativamente do controle (Tabela 4). Aparentemente, a simbiose não estava estabelecida, pois a presença dessas estruturas nas raízes é geralmente considerada como sinal de fun-

Tabela 3. Efeito da desinfestação do solo, da associação com fungos micorrízicos arbusculares e doses de fósforo na porcentagem de colonização total em raízes de mangabeira, 150 dias após a inoculação ${ }^{(1)}$.

\begin{tabular}{|c|c|c|c|c|c|c|}
\hline \multirow[t]{3}{*}{$\mathrm{P}\left(\mathrm{mg} \mathrm{dm}^{-3}\right)$} & \multicolumn{6}{|c|}{ Colonização total (\%) } \\
\hline & \multicolumn{3}{|c|}{ Solo desinfestado } & \multicolumn{3}{|c|}{ Solo não-desinfestado } \\
\hline & G. albida & G. etunicatum & Controle & G. albida & G. etunicatum & Controle \\
\hline $3 *(2)$ & $48,68 \mathrm{aA}$ & $7,85 \mathrm{aB}$ & $0,00 \mathrm{aB}$ & $51,99 \mathrm{aA}$ & $55,515 \mathrm{aA}$ & $52,000 \mathrm{aA}$ \\
\hline 3 & $47,41 \mathrm{aA}$ & $0,89 \mathrm{aB}$ & $0,00 \mathrm{aB}$ & $43,45 \mathrm{abA}$ & $35,852 \mathrm{abcA}$ & $42,127 \mathrm{abA}$ \\
\hline 48 & $14,85 \mathrm{bA}$ & $2,03 \mathrm{aA}$ & $0,00 \mathrm{aA}$ & $43,34 \mathrm{abA}$ & $24,967 \mathrm{bcA}$ & $29,222 \mathrm{abA}$ \\
\hline 93 & $10,17 \mathrm{bA}$ & $1,23 \mathrm{aA}$ & $14,81 \mathrm{aA}$ & $20,38 \mathrm{bcA}$ & $17,537 \mathrm{cA}$ & $32,937 \mathrm{abA}$ \\
\hline 138 & $22,05 \mathrm{bA}$ & $1,37 \mathrm{aB}$ & $0,00 \mathrm{aB}$ & $16,91 \mathrm{cB}$ & $44,050 \mathrm{abA}$ & $24,890 \mathrm{bAB}$ \\
\hline 183 & $9,27 \mathrm{bA}$ & $0,18 \mathrm{aA}$ & $0,00 \mathrm{aA}$ & $37,34 \mathrm{abcA}$ & $28,520 \mathrm{bcA}$ & $33,995 \mathrm{abA}$ \\
\hline Regressão & Cúbica $^{(3)}$ & ns & $\mathrm{ns}$ & Quadrática $^{(4)}$ & Cúbica $^{(5)}$ & Quadrática $^{(6)}$ \\
\hline
\end{tabular}

${ }^{(1)}$ Médias seguidas pela mesma letra, minúsculas na coluna e maiúsculas na linha, não diferem entre si pelo teste de Tukey a 5\% de probabilidade; o coeficiente de variação foi de $32,15 \%$. (2) Isento de solução nutritiva. ${ }^{(3)} \mathrm{y}=53,39^{* *}-1,482^{* *} \mathrm{x}+0,015^{* *} \mathrm{x}^{2}-0,000049^{* * *} \mathrm{x}^{3}\left(\mathrm{R}^{2}=0,98\right)$. ${ }^{(4)} \mathrm{y}=56,37^{* *}$ $-0,49^{*} x+0,0020^{*} x^{2}\left(R^{2}=0,82\right) .{ }^{(5)} y=60,58^{* *}-1,52^{* *} x+0,018^{*} x^{2}-0,00005^{* *} x^{3}\left(R^{2}=0,88\right) .{ }^{(6)} y=50,05^{* *}-0,367^{n s} x+0,016^{n s} x^{2}\left(R^{2}=0,82\right)$.

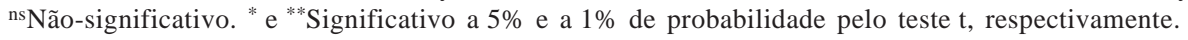

Tabela 4. Efeito da desinfestação do solo e da associação com fungos micorrízicos arbusculares (FMA), independentemente das doses de fósforo, na concentração de fósforo na parte aérea e na porcentagem de arbúsculos e hifas em raízes de mangabeira, 150 dias após a inoculação ${ }^{(1)}$.

\begin{tabular}{|c|c|c|c|c|c|c|}
\hline \multirow[t]{2}{*}{ FMA } & \multicolumn{2}{|c|}{ Fósforo (\% na parte aérea) } & \multicolumn{2}{|c|}{ Arbúsculo (\%) } & \multicolumn{2}{|c|}{ Hifa $(\%)$} \\
\hline & SD & SND & SD & SND & SD & SND \\
\hline G. albida & $0,129 \mathrm{aA}$ & $0,138 \mathrm{aA}$ & $24,05 \mathrm{aB}$ & $33,10 \mathrm{aA}$ & $1,35 \mathrm{aB}$ & $4,16 \mathrm{aA}$ \\
\hline G. etunicatum & $0,008 \mathrm{bB}$ & $0,146 \mathrm{aA}$ & $1,75 \mathrm{bB}$ & $32,02 \mathrm{aA}$ & $0,49 \mathrm{abB}$ & $1,95 \mathrm{bA}$ \\
\hline Controle & $0,008 \mathrm{bB}$ & $0,149 \mathrm{aA}$ & $2,01 \mathrm{bB}$ & $35,89 \mathrm{aA}$ & $0,10 \mathrm{bA}$ & $0,65 \mathrm{cA}$ \\
\hline$\overline{\mathrm{CV}}(\%)$ & \multicolumn{2}{|c|}{36,61} & \multicolumn{2}{|c|}{33,02} & \multicolumn{2}{|c|}{31,38} \\
\hline
\end{tabular}

${ }^{(1)}$ Médias seguidas pela mesma letra, minúsculas na coluna e maiúsculas na linha, não diferem entre si pelo teste de Tukey a 5\% de probabilidade; SD: solo desinfestado; SND: solo não-desinfestado. 
cionamento da associação, e atesta a dinâmica da colonização. Os arbúsculos são considerados sítios de troca por excelência entre os simbiontes e estudos defendem o seu papel na absorção de carbono (Smith \& Read, 1997). Maior quantidade de arbúsculos foi encontrada nas raízes de mangabeira associadas com G. albida (Tabela 4). Em plantas micropropagadas de morango (Fragaria x ananassa Duch cv. Elvira) submetidas à inoculação de nove espécies de FMA, Gigaspora rosea destacou-se por formar a mais alta percentagem por arbúsculos (Taylor \& Harrier, 2001). Os FMA apresentam diferentes estratégias de colonização; segundo Hart \& Reader (2002) isolados de Gigasporaceae apresentam menor taxa de colonização e maior extensão de micélio no solo do que nas raízes, enquanto espécies de Glomaceae produzem maior colonização e extensivo micélio na raiz comparado ao solo. Neste trabalho, G. albida produziu maior colonização total, arbúsculos e hifas do que G. etunicatum, nos tratamentos em solo desinfestado, sendo também mais efetivo em produzir benefícios nutritivos ao hospedeiro.

Maior colonização geralmente é seguida por estímulo no crescimento da planta, na maioria das vezes atribuído ao aumento da nutrição de P, em solo com baixa disponibilidade desse elemento (Smith \& Read, 1997). Em solo desinfestado, a efetividade de G. albida favoreceu a concentração de P na parte aérea (Tabela 4). Resultados semelhantes foram obtidos com bananeiras (Declerck et al., 2002).

Nos tratamentos com a menor dose de $\mathrm{P}$, mudas com G. albida apresentaram maior colonização por arbúsculos em relação aos demais tratamentos de inoculação, mas o aumento no nível de P inibiu a formação dessas estruturas (Tabela 5). Resultados semelhantes, que mostram a redução da colonização micorrízica com a elevação do nível de $\mathrm{P}$, também foram observados em plantas de cafeeiro (Saggin Júnior et al., 1994) e de limoeiro-cravo (Melloni et al., 2000). No tratamento com G. etunicatum, a percentagem de arbúsculos se manteve baixa $(<30 \%)$ mesmo nos tratamentos com reduzidas quantidades de fósforo. No solo sem inoculação a aplicação de $\mathrm{P}$ não influenciou a quantidade de arbúsculos formados pelos FMA nativos (Tabela 5).

A aplicação de brometo de metila como tratamento para desinfestação do solo resulta na eliminação dos fungos micorrízicos nativos. No entanto, estruturas características de FMA foram observadas em alguns tratamentos, o que pode ter sido proveniente de contaminação em casa de vegetação, ou à não-eliminação de alguns propágulos. A reinvasão de FMA que não foram eliminados pode ocorrer entre 2 e 13 meses após a desinfestação do solo com fumigantes químicos (Menge, 1982). Hifas ativas também foram detectadas em experimento com citros, em solo autoclavado e sem adição de FMA (Melloni et al., 2000).

Independentemente do FMA, a mangabeira foi considerada excessivamente dependente da micorrização em solo desinfestado e com $3 \mathrm{mg} \mathrm{dm}^{-3}$ de fósforo. $\mathrm{O}$ grau de dependência variou de acordo com o fungo associado nas mesmas condições de solo, sendo que, nos níveis mais elevados de $\mathrm{P}$ a dependência diminuiu, especialmente no solo desinfestado (Tabela 6). Melloni et al. (2000) relacionaram este comportamento com os valores de micélio externo ativo e total, levando a maior consumo de fotoassimilados e acarretando menor desenvolvimento vegetativo do hospedeiro. Menge et al. (1978) sugeriram outros fatores que podem influenciar a absorção do $\mathrm{P} \mathrm{e}$, conseqüientemente, a dependência micorrízica: presença de pêlos radiculares, taxas de crescimento da planta, transporte e utilização do fósforo. A eficiência da absorção de nutrientes também pode ser afetada por parâmetros de troca como interface fungocélula da raiz (arbúsculos) e extensão, viabilidade e capacidade de transporte da hifa externa (Smith \& Read, 1997).

Tabela 5. Efeito da associação com fungos micorrízicos arbusculares (FMA), independentemente do tratamento de solo, na porcentagem de arbúsculos em raízes de mangabeira, 150 dias após a inoculação, nas diferentes doses de fósforo aplicadas ao solo $^{(1)}$.

\begin{tabular}{llccccc}
\hline FMA & \multicolumn{7}{c}{$\mathrm{P}\left(\mathrm{mg} \mathrm{dm}^{-3}\right)$} \\
\cline { 2 - 7 } & \multicolumn{1}{c}{$3 *(2)$} & 3 & 48 & 93 & 138 & 183 \\
\hline G. albida & $42,62 \mathrm{aAB}$ & $45,96 \mathrm{aA}$ & $25,58 \mathrm{aBC}$ & $13,92 \mathrm{abC}$ & $18,66 \mathrm{aC}$ & $21,13 \mathrm{aC}$ \\
G. etunicatum & $16,37 \mathrm{bAB}$ & $30,33 \mathrm{bA}$ & $3,34 \mathrm{aAB}$ & $8,69 \mathrm{bB}$ & $18,65 \mathrm{aAB}$ & $13,71 \mathrm{aAB}$ \\
Controle & $22,76 \mathrm{bA}$ & $25,59 \mathrm{bA}$ & $16,52 \mathrm{aA}$ & $24,81 \mathrm{aA}$ & $10,66 \mathrm{aA}$ & $11,33 \mathrm{aA}$ \\
\hline
\end{tabular}

(1) Médias seguidas pela mesma letra, minúsculas na coluna e maiúsculas na linha, não diferem entre si pelo teste de Tukey a 5\% de probabilidade; o coeficiente de variação foi de $33,02 \%$. ${ }^{(2)}$ Isento de solução nutritiva. 
Tabela 6. Dependência micorrízica em mudas de mangabeira, 150 dias após a inoculação de fungos micorrízicos arbusculares ${ }^{(1)}$.

\begin{tabular}{cccccc}
\hline $\mathrm{P}\left(\mathrm{mg} \mathrm{dm}^{-3}\right)$ & \multicolumn{2}{c}{ G. albida } & & \multicolumn{2}{c}{ G. etunicatum } \\
\cline { 2 - 3 } \cline { 5 - 6 } & Solo desinfestado & Solo não-desinfestado & & Solo desinfestado & Solo não-desinfestado \\
\hline $3^{*(2)}$ & $94,16 \mathrm{E}$ & $-5,92 \mathrm{I}$ & $85,29 \mathrm{E}$ & $3,24 \mathrm{Ma}$ \\
3 & $86,66 \mathrm{E}$ & $-30,70 \mathrm{I}$ & $59,26 \mathrm{~A}$ & $-16,90 \mathrm{I}$ \\
48 & $4,34 \mathrm{Ma}$ & $27,06 \mathrm{Mo}$ & $8,33 \mathrm{Ma}$ & $-94,00 \mathrm{I}$ \\
93 & $-40,74 \mathrm{I}$ & $37,00 \mathrm{Mo}$ & & $-100,00 \mathrm{I}$ & $46,15 \mathrm{Mo}$ \\
138 & $24,32 \mathrm{Mo}$ & $-92,68 \mathrm{I}$ & $20,00 \mathrm{Ma}$ & $24,76 \mathrm{Ma}$ \\
183 & $-3,95 \mathrm{I}$ & $-41,18 \mathrm{I}$ & $3,57 \mathrm{Ma}$ & $4,95 \mathrm{Ma}$ \\
\hline
\end{tabular}

(1)Dependência micorrízica relativa: $>75 \%=$ excessiva (E); $50 \%-75 \%=$ alta (A); 25\%-50\% = moderada (Mo); <25\% = marginal (Ma); não responde à inoculação = independente (I). ${ }^{(2)}$ Isento de solução nutritiva.

O efeito benéfico da associação micorrízica no crescimento da planta tem sido atribuído, na maior parte das vezes, ao aumento da absorção e da concentração de nutrientes, especialmente o fósforo. A habilidade da planta para absorver $\mathrm{P}$ do solo é considerada o principal fator que contribui para as diferenças observadas na dependência micorrízica relativa de uma espécie (Mosse et al., 1973). Neste trabalho, maior dependência micorrízica em relação aos FMA introduzidos foi observada nos tratamentos em solo desinfestado e com a dose mais baixa de fósforo. A dependência micorrízica da mangabeira variou de acordo com a espécie de FMA, a condição do solo, desinfestado ou não, e com as doses de fósforo. $\mathrm{O}$ mesmo ocorreu em mudas de maracujazeiro-amarelo (Cavalcante et al., 2001). Isso comprova o efeito negativo do aumento da disponibilidade do P na eficiência da simbiose micorrízica (Melloni et al., 2000).

$\mathrm{O}$ menor crescimento das mudas de mangabeira em solo desinfestado e sem inoculação demonstra a alta dependência micorrízica dessa espécie em condições de reduzidas quantidades de $\mathrm{P}$ no solo. Com a desinfestação do solo, a maioria dos microrganismos foi eliminada, inclusive os FMA nativos existentes no solo e que foram eficientes para a mangabeira.

\section{Conclusões}

1. Mudas de mangabeira respondem bem a fungos micorrízicos abusculares nativos na rizosfera, os quais proporcionam aumento no crescimento e maior absorção de $\mathrm{P}$ na parte aérea, em solo natural.

2. Em solo desinfestado, o crescimento de mudas de mangabeira é beneficiado pela associação com G. albida, que é mais efetivo e competitivo do que G. etunicatum no desenvolvimento de mudas de mangabeira.

3. A mangabeira é dependente da micorrização em solo desinfestado e com baixo teor de fósforo $\left(3 \mathrm{mg} \mathrm{dm}^{-3}\right)$.

\section{Agradecimentos}

Ao colega Manoel Bandeira de Albuquerque (UFRPE), pelo fornecimento das sementes de mangabeira e apoio na coleta em campo; ao Dr. Everardo Sampaio (UFPE), pela colaboração em relação à fertilização do solo; à Capes, pela bolsa concedida a Cynthia Maria Carneiro Costa; ao CNPq, pelo apoio financeiro e concessão de bolsa de pesquisa a Leonor Costa Maia.

\section{Referências}

AGUIAR FILHO, S.P.; BOSCO, J.; ARAÚJO, I.A. A mangabeira (Hancornia speciosa): domesticação e técnicas de cultivo. João Pessoa: Emepa-PB, 1998. 26p. (Documentos, 24).

AMIJEE, F.; TINKER, P.B.; STRIBLEY, D.P. The development of endomycorrhizal roots systems. New Phytologist, v.111, p.435446, 1989.

CAVALCANTE, U.M.T.; MAIA, L.C.; COSTA, C.M.C.; SANTOS, V.F. Mycorrhizal dependency of passion fruit (Passiflora edulis $\mathrm{f}$. flavicarpa). Fruits, v.56, p.317-324, 2001.

CAVALCANTE, U.M.T.; MAIA, L.C.; COSTA, C.M.C.; CAVALCANTE, A.T.; SANTOS, V.F. Efeito de fungos micorrízicos arbusculares, da adubação fosfatada e da esterilização do solo no crescimento de mudas de maracujazeiro amarelo. Revista Brasileira de Ciência do Solo, v.26, p.1099-1106, 2002a.

CAVALCANTE, U.M.T.; MAIA, L.C.; MELO, A.M.M.; SANTOS, V.F. Influência da densidade de fungos micorrízicos arbusculares na produção de mudas de maracujazeiro-amarelo. Pesquisa Agropecuária Brasileira, v.37, p.634-649, 2002b.

CHU, E.Y.; MÖLLER, M.R.F.; CARVALHO, J.G. Efeitos da inoculação micorrízica em mudas de gravioleira em solo fumigado e não fumigado. Pesquisa Agropecuária Brasileira, v.36, n.4, p.671680, 2001.

CLAPPERTON, M.J.; REID, D.M. A relationship between plant growth and increasing VA mycorrhizal inoculum density. New Phytologist, v.120, p.227-234, 1992.

COSTA, C.M.C.; MAIA, L.C.; CAVALCANTE, U.M.T.; NOGUEIRA, R.J.M.C. Influência de fungos micorrízicos 
arbusculares sobre o crescimento de dois genótipos de aceroleira (Malpighia emarginata D.C.). Pesquisa Agropecuária Brasileira, v.36, p.893-901, 2001.

DECLERCK, S.; RISEDE, J-M.; DELVAUX, B. Greenhouse response of micropropagated bananas inoculated with in vitro monoxenically produced arbuscular mycorrhizal fungi. Scientia Horticulturae, v.93, p.301-309, 2002.

DONADIO, L.C.; MÔRO, F.V.; SERVIDONE, A.A. Frutas Brasileiras. Jaboticabal: Novos Talentos, 2002. 288p.

EDIGITON, L.V.; KHEW, K.L.; BARRON, G.L. Fungitoxic spectrum of benzimidazole compounds. Phytopathology, v.61, p.4244, 1971.

GERDEMANN, J.W.; NICOLSON, T.H. Spores of mycorrhizal Endogone species extracted from soil by wet sieving and decanting. Transactions of the British Mycological Society, v.46, p.235244, 1963.

HABTE, M.; MANJUNATH, A. Categories of vesicular-arbuscular mycorrhizal dependency of host species. Mycorrhiza, v.1, p.3-12, 1991.

HART, M.M.; READER, R.J. Taxonomic basis for variation in the colonization strategy of arbuscular mycorrhizal fungi. New Phytologist, v.153, p.335-344, 2002.

JENKINS, W.R. A rapid centrifugal flotation technique for separating nematodes from soil. Plant Disease Reporter, v.48, p.692, 1964.

JORGE, L.A.C.; CAMPOS NETO, D.; LEONI, E.; CRESTANA, S. Aquisição de imagens de folhas. In: JORGE, L.A.C. (Ed.). Recomendações práticas para aquisição de imagens digitais analisadas através do SIARCS ${ }^{\circledR}$. São Carlos: Embrapa-CNPDIA, 1996. p.40-56. (Circular Técnica, 1).

LINS, G.M.L.; TRINDADE, A.V.; ROCHA, H.S. Utilização de Gigaspora margarita em plantas micropropagadas de bananeira em diferentes estádios de enraizamento. Revista Brasileira de Fruticultura, v.25, p.143-147, 2003.

MALAVOLTA, E. Avaliação do estado nutricional das plantas: princípios e aplicações. Piracicaba: Associação Brasileira para Pesquisa da Potassa e do Fosfato, 1989. 201p.

McGONIGLE, T.P.; MILLER, M.H.; EVANS, D.G.; FAIRCHILD, G.L.; SWAN, J.A. A new method which gives an objective measure of colonization of roots by vesicular-arbuscular mycorrhizal fungi. New Phytologist, v.115, p.495-501, 1990.

MELLONI, R.; NOGUEIRA, M.A.; FREIRE, V.F.; CARDOSO, E.J.B.N. Fósforo adicionado e fungos micorrízicos arbusculares no crescimento e nutrição mineral de limoeiro-cravo [Citrus limonia (L). Osbeck]. Revista Brasileira de Ciência do Solo, v.24, p.767775,2000

MENGE, J.A. Effect of soil fumigant and fungicides on vesiculararbuscular fungi. Phytopathology, v.72, p.1125-1182, 1982.
MENGE, J.A.; JOHNSON, E.L.V.; PLATT, R.G. Mycorrhizal dependency of several citrus cultivars under three nutrient regimes. New Phytologist, v.81, p.553-559, 1978.

MIRANDA, J.C.C.; MIRANDA, L.N. Micorriza arbuscular. In: VARGAS, M.A.T.; HUNGRIA, M. (Ed.). Biologia dos solos dos Cerrados. Planaltina: Embrapa-CPAC. 1997. p.67-123.

MOSSE, B.; HAYMAN, D.S.; ARNOLD, D.J. Plant growth response to vesicular-arbuscular mycorrhiza. V. Phosphate uptake by three plant species from P-deficient soils labeled with ${ }^{32} \mathrm{P}$. New Phytologist, v.72, p.809-815, 1973.

PHILLIPS, J.M.; HAYMAN, D.S. Improved procedures for clearing roots and staining parasitic and vesicular arbuscular mycorrhizal fungi for rapid assessement of infections. Transactions of the British Mycological Society, v.55, p.158-161, 1970.

PLENCHETTE, C.; FORTIN, J.A.; FURLAN, V. Growth responses of several plant species to mycorrhizae in a soil of moderate Pfertility. I. Mycorrhizal dependence under field conditions. Plant and Soil, v.70, p.199-209, 1983.

SAGGIN JUNIOR, O.J.; SIQUEIRA, J.O.; GUIMARÃES, P.T.G.; OLIVEIRA, E. Interação fungos micorrízicos versus superfosfato e seus efeitos no crescimento e teores de nutrientes do cafeeiro em solo não fumigado. Revista Brasileira de Ciência do Solo, v.18, p.27-36, 1994.

SAMARÃO, S.S.; MARTINS, M.A. Influência de fungos micorrízicos arbusculares, associadas à aplicação de rutina, no crescimento de mudas de goiabeira (Psidium guajava L.). Revista Brasileira de Fruticultura, v.21, p.196-199, 1999.

SCHENCK, N.C.; PÉREZ, Y. Manual for the identification of VA mycorrhizal fungi. 3.ed. Gainesville: Synergistic Publ., 1990.

SMITH, S.E.; READ, D.J. Mycorrhizal Symbiosis. London: Academic Press, 1997. p.453-469.

TAYLOR, J.; HARRIER, L.A. A comparison of development and mineral nutrition of micropropagated Fragaria $\mathrm{x}$ ananassa cv. Elvira (strawberry) when colonized by nine species of arbuscular mycorrhizal fungi. Applied Soil Ecology, v.18, p.205-215, 2001.

TRINDADE, A.V.; SIQUEIRA, J.O.; ALMEIDA, F.P. Eficiência simbiótica de fungos micorrízicos arbusculares em solo não fumigado, para mamoeiro. Revista Brasileira de Ciência do Solo, v.24, p.505$513,2000$.

WEST VIRGINIA UNIVERSITY (Morgantown, Estados Unidos). INVAM: international culture collection of (vesicular) arbuscular mycorrhizal fungi. Disponível em: $<$ http: // invam. caf . wvu. edu $>$. Acesso em: fev. 2003.

WILSON, J.M.; TOMMERUP, I.C. Interactions between fungal symbionts: VA mycorrhizae. In: ALLEN, M.F. (Ed.). Mycorrhizal functioning. London: Chapman and Hall, 1992. p.199-248.

Recebido em 8 de abril de 2004 e aprovado em 28 de dezembro de 2004 